\title{
The integration of information systems in a medical facility to improve the efficiency of decision-making
}

\author{
Marina Bolsunovskaya ${ }^{1}$, Alexander Leksashov $^{1}$, Aleksey Gintciak $^{1}$, and Anastasiia \\ Shirokova ${ }^{*}$ \\ ${ }^{1}$ Peter the Great St Petersburg Polytechnic University, St Petersburg, Russia
}

\begin{abstract}
This paper describes a project for integrating a medical and diagnostic information system in a medical facility. At the moment, making effective and timely decisions in diagnosis and treatment is most important. Integration of systems has significantly improved the efficiency of decision-making by updating data. The unified information space allowed to increase the objectivity of diagnostics and increase the reliability of the information system. A critical factor in making a decision is the stage at which the disease is detected, as well as a description of what types of medical care are important for analysis. In addition, the need to synchronize on the basis of the detailed information for operational planning and routing of patients. Integration of solutions will increase the speed of passing examinations and the speed of making a final diagnosis. Thanks to the implemented solution, it became possible to increase the speed and improve the quality of services provided. The ability to synchronize activity planning based on effective diagnostics is essential.
\end{abstract}

\section{Introduction}

In order to improve the efficiency of decision-making in the diagnosis and treatment of diseases, it was decided to integrate accounting and diagnostic information systems in a medical institution. In addition to basic information, the integrated system should store and update in a timely manner information that characterizes the flow of patients according to the severity of the disease, the characteristics of the hospitalization process, the characteristics of the bed stock, and the workload of beds.

In modern conditions of medical care, the use of advanced technologies and equipment has a huge impact. The use of diagnostic equipment allows you to detect the disease in a timely manner and start its treatment at an early stage. At the moment, equipment for computed tomography and radiology is of particular importance. In addition, diagnostics require integration of hardware and software into a single hardware and software package. This makes it possible to create a single information space. It also significantly accelerates decision-making based on data received from diagnostic equipment. To achieve this goal, the medical institution implements the integration of medical information systems. The

* Corresponding author: stasja_c@ mail.ru 
medical information system (MIS) and diagnostic information system (DIS) are being integrated.

Historically, the MIS and DIS systems were developed separately in a medical institution. MIS is a complex, integrated information system designed to support work in a medical institution, in terms of medical, administrative, financial and legal issues, as well as services provided. DIS is a system for working with images. DIS provides storage and timely access to diagnostic studies. To provide high-quality, comprehensive services, it is necessary to ensure timely, automatic data exchange between information systems [1].

The authors analyzed existing information systems and described existing ("AS-IS") business processes for providing medical care to patients. The process of data exchange between the considered information systems is also analyzed. The rationale for the integration process, the choice of the router program, configuration and installation of the selected solution. The effectiveness of the integration solution (integration platform) was evaluated.

\section{Justification of the need to integrate medical and diagnostic information systems}

MIS is part of the whole complex of Informatization in the institution. The main focus of the MIS is to meet the administrative needs of the medical complex.

The basic version of the program is installed in the medical institution, and additional modules are also included (electronic patient history, data uploading for accounting systems) [2-3].

The basic version performs the following main functions:

* maintaining a patient file with all the necessary information about the patient, control of filling in fields, search capability;

* ability to issue documentation for the patient;

* maintenance of work schedules, schedules for the reception of specialists;

- use of reference books for diagnosis, quick and convenient input of information;

- a system for managing payments with patients, with the ability to issue invoices and payment history;

* organization of work with the patient queue, formation of waiting lists.

In addition, there is a module "Electronic patient history". This module allows you to get more detailed information about the patient, in addition to personal data, the doctor can get or fill in the following information:

- anamnesis of life (allergies, bad habits, previous illnesses, injuries, etc.) •

* history of previous research, in particular clinical and diagnostic.

The doctor can independently configure templates for the history of diseases, use ICD 10 reference books, reference books of medicines, view and copy any previously filled information from the protocols, and automatically receive data from laboratory analyzers.

The center also has the module "Uploading data for accounting systems".

As part of the project, the authors are developing the following improvements to the basic version of the medical information system:

1. Characteristics of the flow of patients according to the severity of the disease, namely:

- the number of new patients who recovered and died, people/day;

- the proportion of new patients in a light condition, in a moderate condition, in a serious condition, $\%$;

- the number and name of the carried out diagnostic studies, and pieces for the day.

2. Characteristics of the process of hospitalization of patients: 
- the proportion of new patients to be hospitalized in relation to the total number of new patients in terms of severity of the disease, $\%$;

- the average duration of hospitalization of patients for each of the degrees of severity of the disease, days.

3. Characteristics of special features of the medical institution:

- the number of available prepared and certified beds in infectious diseases departments, intensive care units, intensive care units, including those with ventilators for all institutions that receive patients with suspected COVID-19 and community-acquired pneumonia, units;

- the number and timing of repurposed or newly prepared and certified beds planned for commissioning in infectious diseases departments, intensive care units, and intensive care units, including those with ventilators, for all institutions that receive patients with suspected COVID-19 and community-acquired pneumonia.

The concept of RIS (radiological information systems) combines all the components of a modern x-ray Department [4]. The RIS system can be divided into several main components:

1. Modalities-diagnostic devices such as computer (CT) and magnetic resonance (MRI) tomography scanners, digital $\mathrm{x}$-ray machines, ultrasound machines, etc. The term "modality" in the context of digital radiology also means the type of study, depending on the principle of operation of the device on which it is made. For example, studies performed on an MRI machine belong to the "MR" modality, digital $\mathrm{x}$ - rays belong to "CR", and $\mathrm{x}$ ray mammography images belong to "MG".

Structurally, the diagnostic device can be divided into three main components, in addition to auxiliary units, such as power cabinets and climate control units-a data collection system that implements the physical principles of the study, a reconstruction system that converts analog or digital signals (raw data) received from the data collection system, and an operator console that serves as an interface for the $\mathrm{x}$ - ray laboratory technician or doctor, and also performs the functions of controlling and controlling equipment in the device. Depending on the type of device, the reconstruction system and the operator console can be combined in one computer or server [5].

2. DIS-a system for collecting, storing and processing research obtained on modalities. The DIS system can also take part in managing the workflow of the x-ray Department, provide access to data to clinicians, and participate in data exchange with systems of other departments. DIS systems are focused on providing trouble-free reception, reliable data storage and providing a basic universal set of tools for working with them. The two main components of DIS systems have always been a productive DBMS and a reliable storage system, most often focused on storing two or more copies of files on different physical servers, preferably located in different rooms or buildings [6,7]. The application server is also implemented separately, which provides communication with the modalities and workplaces of doctors.

3. Systems for special and expert visualization and processing of research data $[8,9]$. This type of information systems includes specialized workstations for various diagnostic techniques, for example, workstations for doctors of computed tomography or mammography, which are used to automate calculations, identify pathologies and other tasks facing radiologists in a particular field. In these systems, automated applications based on image recognition are widely used to perform routine tasks and perform standardized calculations.

Historically, such applications were separated into a separate type of CAD systems (computer aided diagnostics) due to the specificity and resource intensity of applications, but today CAD applications are part of expert visualization systems. Often, large manufacturers of medical equipment combine tools for working with data from various 
modalities in one system, creating a basic platform with a single user interface, supplemented by the necessary modules for a specific medical institution. Such systems usually have a client-server architecture and are based on servers with large computing resources [10. 11]. However, due to the fact that these systems are not focused on troublefree data storage, which is provided by the DIS system, they are equipped with smaller storage systems in terms of the volume of stored data, sufficient for operational storage of data for a short period.

When organizing interaction between MIS and DIS, you can significantly speed up the interaction processes. In addition, this will avoid erroneous patient data entry and a significant amount of paperwork:

* the Clinician makes a request for research through the MIS;

- the Registrar of the X-ray Department selects the time for the study in the MIS office, the patient's data is already in the MIS;

* the patient is registered on the device console before the study;

* the description of the study is made in the MIS and available to the Clinician immediately;

- in addition to the description, the Clinician can view the images via the DIS client app.

After integration of MIS and DIS systems, all documents necessary for high-quality patient care will be transferred between the systems automatically. In other words, the patient will not pick up the results of tests on their own, there will be no need to print out directions and conclusions, and all documents will be automatically visible in the systems. You will also be able to record a patient to the right specialist without contacting the registry, directly from the synchronized system.

There is no paperwork, there is no possibility of entering demographic data incorrectly, and the unique patient index is end-to-end in all systems.

The authors describe and analyze the business process of hospitalization of a patient. Based on the analysis, the weaknesses of the existing process were identified. After defining the boundaries and "areas of responsibility" between the systems, an analysis of existing solutions was carried out. In order to make an informed choice, the decisionmaking methodology was divided into stages. The selection was made in several steps with the calculation of intermediate results. In order to make the decision as reasonable as possible, a team representing various areas of activity was assembled. All the steps were completed, starting from the formation of requirements for the integrator, taking into account possible errors within a specific medical institution, ending with the final selection between several vendors. Thus, the base was prepared for the implementation of the integrator implementation project.

In order to successfully implement the integration, the task statement was worked out in detail. The information flows that the systems will exchange were analyzed, the main standards for data transfer between medical systems were considered, and the technical aspect of the project implementation was also touched upon. After the task was formulated, the applied part of the work was performed. In order to implement the integration of MIS and DIS [12], after preliminary preparation, the Mirth Connect platform was installed on the computer that serves as the server [13]. After the installation has been carried out the process of setting up channels, which provided a correct re-transfer messages, and configuration mapping, this process in turn provided the correct conversion of messages. In order for all data to be transmitted correctly, to ensure that information can be transmitted and changed in a timely manner in the two systems, a script was written that sets the rules of operation. The result of this work is correctly functioning and synced MIS and DIS systems, data between which is transmitted automatically [14]. 


\section{Results}

The result of this work is correctly functioning and synchronized MIS and DIS systems, data between which is transmitted automatically. Both the functional and technical components of the project were implemented [15]. Integration made it possible to ensure timely and effective decision-making by the staff of the medical institution. And this, in the present conditions, is extremely important for improving medical care.

The authors evaluated the qualitative effect of the integration of information systems in a medical institution. Indicators that measure the results achieved and indicators that reflect the processes that contribute to achieving these results were evaluated. When evaluating, both categories of indicators should be linked to each other, since in order to achieve the first (for example, a certain level of productivity), it is necessary to implement the second (for example, to achieve a certain capacity utilization of machines and equipment).

Let's highlight the indicators that reflect the most important indicators for a medical institution:

1. Increase in the number of examined patients. By significantly saving time spent on re-entering data, it is possible to increase the number of studies.

2. Increase the speed of research. Due to the fact that the staff will spend less time on routine operations, the speed of research will increase and the waiting time in the queue will be reduced.

3. Increase the effective operating time of the equipment. Synchro-nization of systems eliminates the need to re-enter information, and the equipment works more efficiently.

4. Improving the quality of services provided. By synchronizing the systems, it is possible to redistribute the doctor's time. now it is not spent on routine operations, but can be spent on a more detailed analysis of the data received from the devices

5. effective diagnosis and decision-making based on complete and adequate information about the current situation and opportunities significantly increases the effectiveness of measures taken and, ultimately, treatment.

6. The training of employees. Due to significant time savings during the year, it becomes possible to release staff for the purpose of training and professional development.

\section{Conclusion}

As a result of implementing the chosen solution based on modern technologies, it was possible to integrate medical information systems. This allowed us to implement:

1. Informing the DIS system about the appointment of new studies, followed by the formation of a working list for diagnostic devices. This makes it possible not only to enter information about planned studies into the device's work schedule, but also to transmit demographic and patient identification data to the DIS, which makes it easier to search for a patient in the database and eliminates input errors.

2. Informing the DIS system about changes in the patient's demographic or identification data. If, due to an input error or other circumstances, the patient's last name or date of birth must be changed in the MIS database, these data will be changed automatically in the DIS.

3. No need to change the code and settings of the MIS and DIS systems. Settings for sending and changing messages are made on an independent platform.

The research is partially funded by the Ministry of Science and Higher Education of the Russian Federation as part of World-class Research Center program: Advanced Digital Technologies (contract No. 075-15-2020-934). 


\section{References}

1. O.G. Kantor, S.I. Spivak, Informatics and its application [Informatika i ee primenenie], 8(2), 111-121(2014)

2. V.N. Volkova Theory of information systems. Textbook [Teoriya informacionnykh sistem. Uchebnoe posobie] (St. Petersburg: Saint-Petersburg Polytechnic University Publ., 300, 2014)

3. V.N. Volkova V. N. Kozlov, V.E. Mager, L.V. Chernenkaya, Proc. 20th IEEE International Conference on Soft Computing and Measurements SCM-2017 (St. Petersburg: ETU “LETI") 183-186 (2017)

4. R. Choplin, Picture archiving and communication systems: an overview (R. Choplin, Radiographics, New York, 127-129, 1992)

5. J. Traeger, OTB Solutions Vendor Selection. The thirteen steps of highly successful HL7 engine selections (J. Traeger, OTB Solutions, New York, 80, 2013)

6. M. Bolsunovskaya, S. Shirokova, A. Loginova, E3S Web of Conferences, 135, 04076 (2019)

7. M.V. Bolsunovskaya, S.V. Shirokova, A.V. Loginova, M.B. Uspenskiy, IOP Conference Series: Materials Science and Engineering, 940(1), 012010 (2020)

8. INFOLine, Research INFOLine Retail Russia TOP-100. // Official site INFOLine, https://infoline.spb.ru/shop/issledovaniya-rynkov/page.php

9. Core Health Technologies, HL7 Interface Technology Survey Results/ Official site, http://corehealthtechnologies.com/main/wp-content/uploads/2012/10/2012-HL7Interface-Technology-Survey-Results.pdf

10. A.A. Denisov, Introduction to information systems analysis (LPI, Leningrad, 67, 1980)

11. A.A. Denisov, Modern problems of system analysis [Sovremennye problemy systemnogo analiza] $(293,2008)$

12. Agfa Healthcare, HL7 Conformance Statement/ Official site, http://www.agfahealthcare.com/global/en/main/products_services/productinfo/interoperability/hl7/index.jsp

13. Mirth, Introduction to Mirth connection/ Official site, https://www.mirth.com/Products-and-Services/Mirth-Connect

14. V.N. Volkova, Systems theory and systems analysis in the management of organizations [Teoriya system i sistemnyi analiz v upravlenii organizatsiyami] (ed. V.N. Volkova, A.A. Emelyanov, Moscow, 847, 2013)

15. M. Yu. Ermolenko: Integrated business planning as minimization of uncertainty through multi-level planning (Establishment of the Russian Academy of Sciences V. A. Trapeznikov Institute of management problems of the Russian Academy of Sciences. Under the General editorship of S. N. Vasiliev, A. D. Tsvirkun, 266-267, 2009) URL: https://elibrary.ru/item.asp?id=26325728 